\title{
MECHANISM OF ARTERIAL PRESSURE OSCILLATIONS CAUSED BY COMPRESSING THE CEREBRAL ARTERY
}

\author{
Kiichi SAGAWA* \\ Department of Physiology, Yokohama University \\ School of Medicine
}

In 1955 MiYAKAWA reported a new method to cause sustained arterial pressure oscillations in rabbits, which consisted in applying an isotonic pressure to the outside of one carotid artery while interrupting all other routes of entry of arterial blood into the brain ${ }^{1)}$. Because the blood pressure oscillations appeared without fail whenever this outside pressure exceeded the systemic arterial pressure, MiYAKAWA pointed out the prime importance of the acute, intense pressor response of the arterial pressure to cerebral ischemia in the genesis of this periodic phenomenon.

Concerning the cerebral ischemic pressor response, the author et al. have recently performed mathematical analyses based on modern control theory and disclosed the highly unstable nature of it as a negative feedback control mechanism ${ }^{2-4)}$. Accordingly, it seemed to the author to be an inevitable forward step to reinvestigate MrYAKAWA's experiment in dogs so that the mechanism of arterial pressure oscillations under the circumstance might be well explained in the light of servoanalytic findings previously obtained on the above mentioned response in the same animal.

\section{METHOD}

In five dogs anesthetized with'sodium pentobarbital $(30 \mathrm{mg} / \mathrm{kg})$, a surgical operation could be performed so that the arterial blood supply, to the brain would be restricted solely to that through the right common carotid artery. The left carotid artery, the vertebral arteries, the anterior spinal artery, and all other arteries through the neck muscles were completely dissected and ligated at the level of the first cervical vertebra. Details of this surgery were reported elsewhere ${ }^{5}$. Here, it should be emphasized that completeness of isolation of the cerebral arterial blood supply from the systemic arterial system was an essential key to the successful experiment.

For the purpose of loading a side pressure of isotonic character upon the one remaining carotid artery, the artery was sectioned and a Starling's resistance was inserted between the cut ends as illustrated in FIG. 1. The resistance consisted of an extremely thin rubber tubing (cigarette tubing) which would easily collapse as soon as the ex-

Received for publication July 21, 1962.

* 佐川喜一 


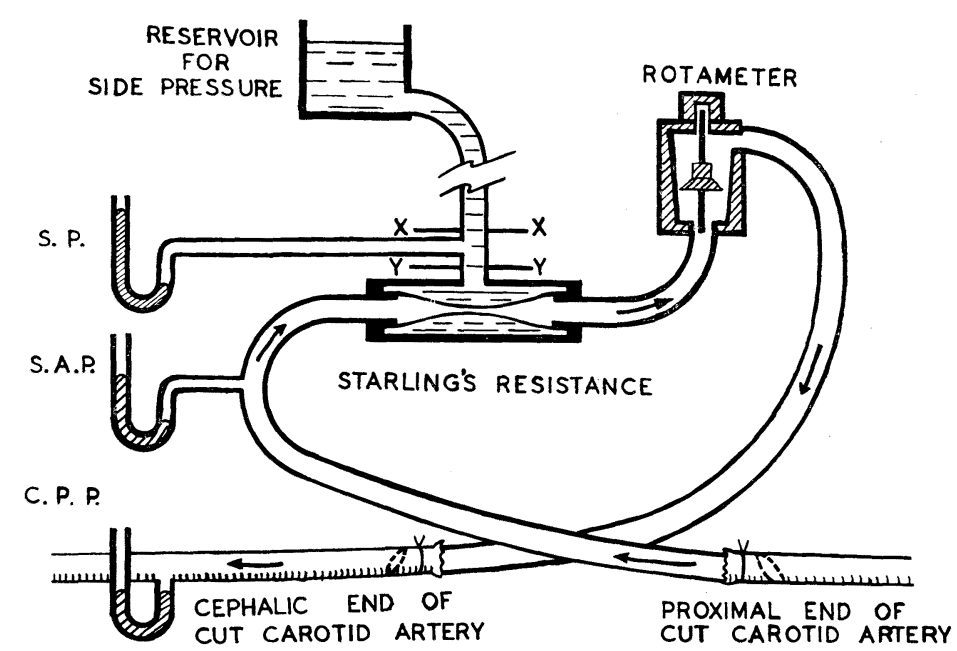

FIG. 1. Illustration of Starling's resistance inserted in the common carotid artery and of the side pressure loading system. The lines $\mathrm{X}-\mathrm{X}$ and $\mathrm{Y}-\mathrm{Y}$ indicate the points at which the rubber tubing was clamped to decrease the compliance of the side pressure loading system.

ternal pressure exceeds the internal pressure, and of a plastic chamber which surrounded the former and was filled with water from a reservoir bottle. By placing this bottle at various hydrostatic levels in relation to that of the chamber, a pressure of desired magnitude could be generated around the collapsible tubing. This pressure was recorded kymographically by a mercury manometer simultaneously with the dog's systemic arterial pressure measured at the femoral artery.

A third mercury manometer recorded the downstream pressure across the Starling's resistance so that the effective cerebral perfusion pressure might be continuously compared with the systemic arterial pressure. Also recorded was the blood flow to the brain through the resistance by means of a Shipley-Wilson type rotameter and a Grass polygraph, which recorded also the above mentioned three kinds of pressures in order to give a more accurate information on the tempcral relationship among these quantities than the kymographic recording.

The nonlinear character of the Starling's resistance used in the present experiment is shown in Fig. 2. When the upstream pressure of the resistance was raised from zero to $180 \mathrm{~mm} \mathrm{Hg}$ with a side pressure fixed at $120 \mathrm{~mm} \mathrm{Hg}$, the flow volume shows little increase until the upstream pressure reaches $120 \mathrm{~mm} \mathrm{Hg}$, but thereafter it suddenly increases close to volumes which would be expected under the corresponding perfusion pressures with zero side pressure as indicated by the broken line in the figure. From this curve it will be understood that the cerebral blood flow is substantially cut off whenever the systemic arterial pressure falls below the side pressure, while when the former pressure surpasses the latter, the effective perfusion pressure to determine the flow volume equals not the difference between the two pressures but approximately the former pressure itself. Such a prominent nonlinearity as seen in FIG. 2 appeared only after both longitudinal edges of the cigarette skin tubing were clipped as long as $2 \mathrm{~cm}$ so that it would be completely collapsed by a very slight inner and outer pressure difference. 


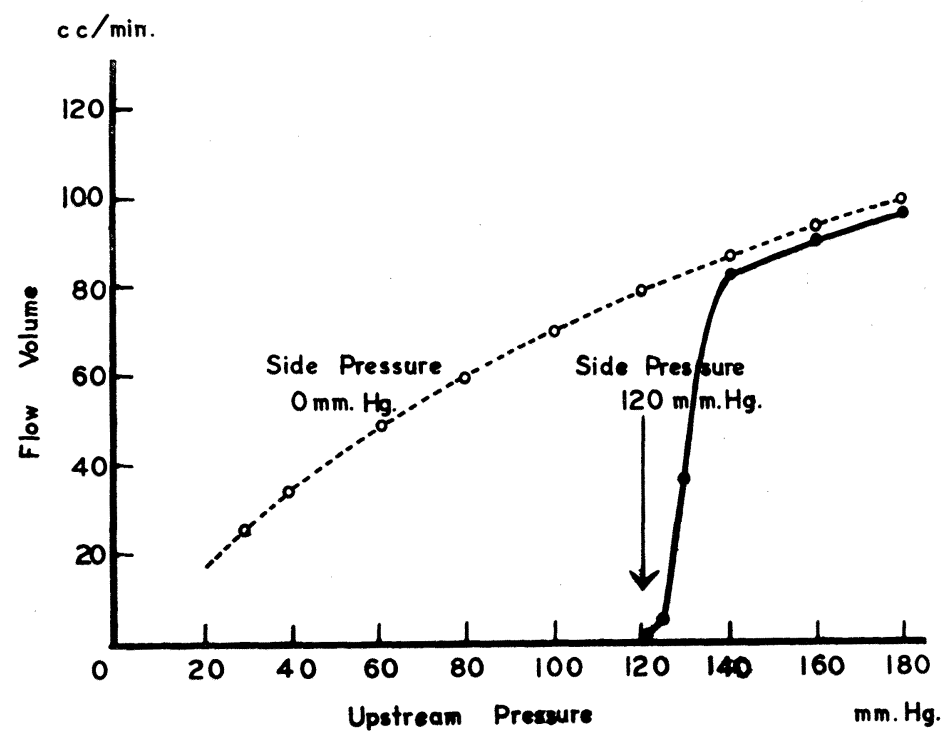

FIG. 2. Pressure-flow relationships through the Starling's resistance illustrated in Fig. 1 with major flow impedance given at a point distal to the resistance by means of a screw clamp. The solid line indicates the relationship when the side pressure was fixed at $120 \mathrm{~mm} \mathrm{Hg}$ and the upstream pressure was altered from zero to $180 \mathrm{~mm} \mathrm{Hg}$, while the broken line shows when the side pressure was zero.

\section{RESULTS}

1. Elicitation of systemic arterial pressure oscillation. In all dogs the side pressure (SP) was elevated stepwise from zero to $140 \mathrm{~mm} \mathrm{Hg}$ with resultant elicitation of systemic arterial pressure (SAP) oscillations when the side pressure reached a certain critical magnitude. FIG. 3 is one example of the kymographic recordings showing the onset of such oscillatory state. In this instance, the oscillation was initiated with the SP set at $80 \mathrm{~mm} \mathrm{Hg}$; the average period and amplitude of three waves recorded there were 60 seconds and $20 \mathrm{~mm} \mathrm{Hg}$ respectively. By further elevation of the SP at $100 \mathrm{~mm} \mathrm{Hg}$, the period and amplitude slightly increased to 63 seconds and $40 \mathrm{~mm} \mathrm{Hg}$ respectively, with simultaneous increase of the mean pressure level around which the SAP oscillated from $85 \mathrm{~mm} \mathrm{Hg}$ to $110 \mathrm{~mm} \mathrm{Hg}$.

Essentially the same response was observed in all other cases, though the average period and amplitude of evoked oscillations in each instance ranged from 21.5 to 63 seconds and from 10 to $100 \mathrm{~mm} \mathrm{Hg}$, respectively. However, a much more important finding evidenced in FIG. 3 is that, so long as the SAP oscillations continue, the cerebral perfusion pressure (CPP) undulates with much larger amplitudes. This is principally due to abrupt fall or rise of the latter pressure as the SAP yields or exceeds the SP. The nonlinear character 


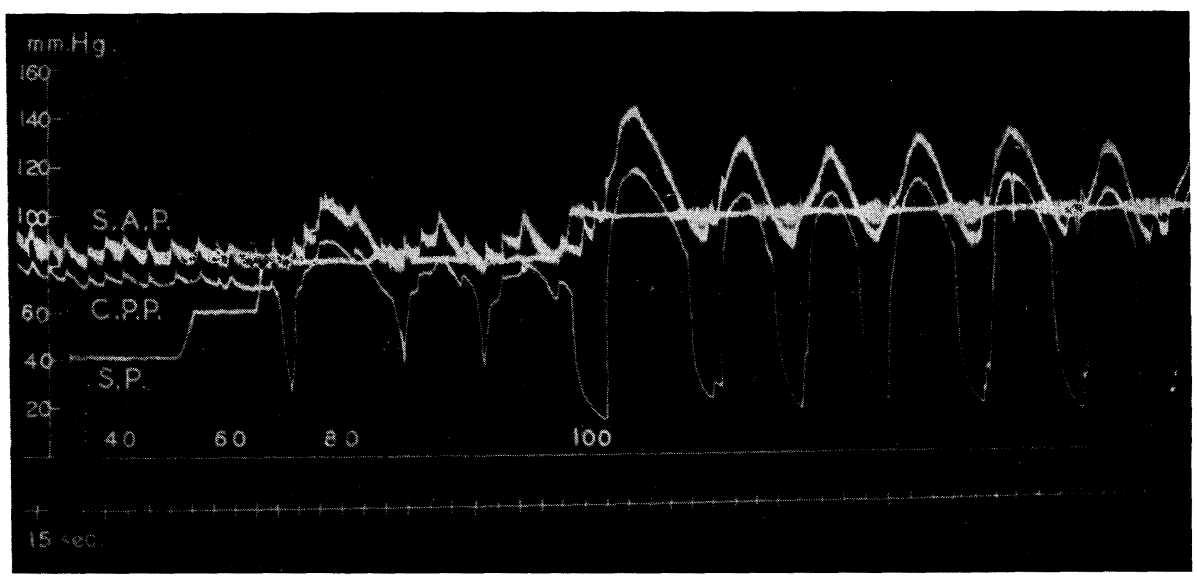

FIG. 3. Kymographic recording of the systemic arterial pressure (SAP), the cerebral perfusion pressure (CPP), and the side pressure (SP) within the Starling's resistance chamber as illustrated in FIG. 1. The SP was raised stepwise from zero to $40,60,80$, and $100 \mathrm{~mm} \mathrm{Hg}$. With the latter two side pressures, the SAP lapsed into a sustained oscillation. Note the exponential fall or the acute rise of the CPP after the SAP succumbed to or overcame the SP, which results in larger amplitude of CPP oscillation than that of SAP. Time signal: 15 seconds.

of the Starling's resistance illustrated in FIG. 2 will explain clearly why such a discrepancy arises between the magnitudes of changing components of these two pressures. As the SAP reaches the SP, the "cigarette skin" tubing in the Starling's resistance chamber closes and the cerebral perfusion pressure distal to the resistance falls exponentially from a pressure level near the SAP toward a pressure close to zero.

During this closure period an increasingly severe degree of ischemia builds up in the brain, which in turn elicits pressor response in the SAP control system after a fairly long time lag. As this pressor response grows intense enough to raise the SAP above the SP, the resistance opens up and the cerebral flow resumes as evidenced by the flow meter record in FIG. 4, or also by the abruptly rising CPP record in FIG. 3. During the following patent period, the pre-existant ischemia and the ischemic response rapidly die away. This results in the fall of SAP, and the Starling's resistance closes again. Thus, the resistance functions here as a sort of pressure limiter for the CPP, which consequently causes nonlinear amplification of the time dependent components of the SAP. The degree of apparent amplification (or gain) by this nonlinear transfer element is a complex function of various physiological factors such as compliance and resistance of the cerebral vascular conductivity while the CPP is falling, and also of the intensity of and the dead time for the cerebral ischemic pressor response while the CPP is rising. The actual degree of such amplification was found to be in the range of 2 to 12 within the present ex- 


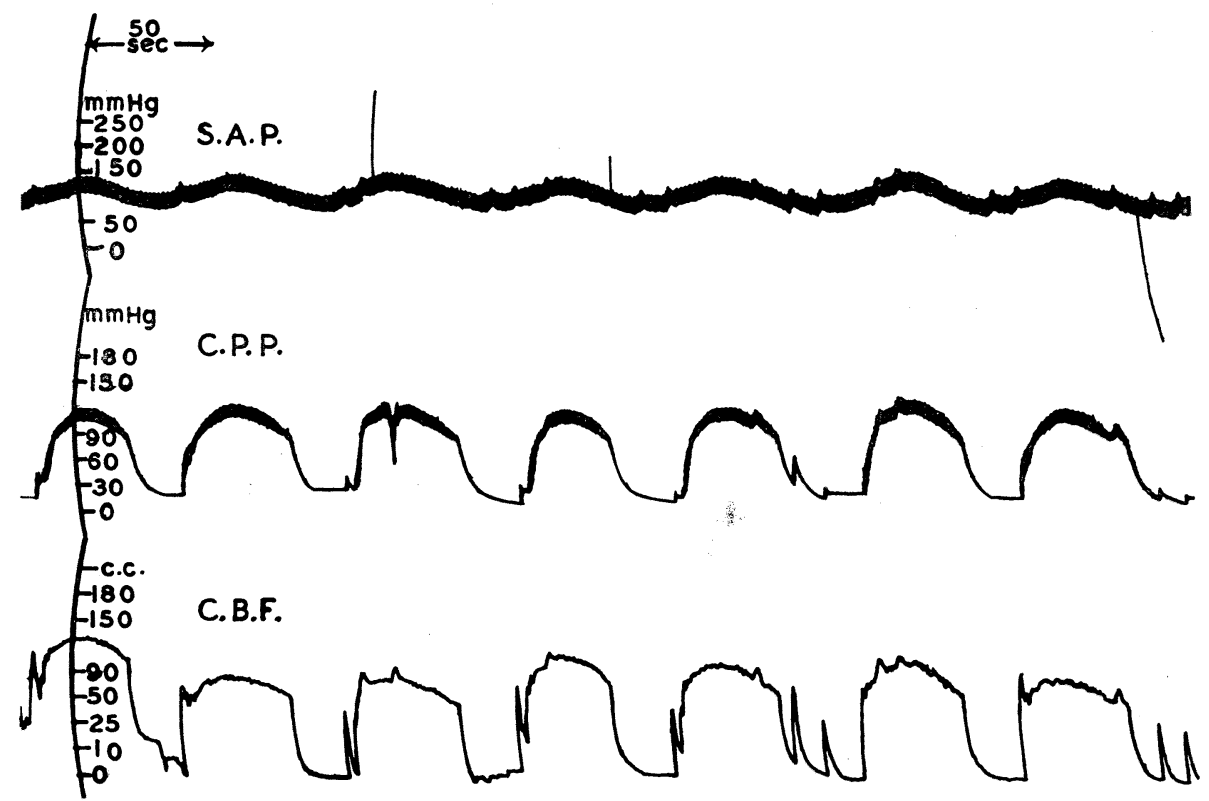

Fig. 4. Polygraphic recording of the SAP, CPP and the cerebral blood flow (CBF), taken simultaneously with the kymographic recording in FIG. 3 while the $\mathrm{SP}$ was set at $100 \mathrm{~mm} \mathrm{Hg}$.

perimental scope.

2. Influence of changing the compliance of the side pressure system. MIYAKAYA checked the influence of altering the compliance of the SP loading system by clamping the rubber tubing of the system at various points as shown by $\mathrm{X}-\mathrm{X}$ and $\mathrm{Y}-\mathrm{Y}$ in Fig. 1.

When the point $\mathrm{X}-\mathrm{X}$ was clamped, the compliance of the side pressure adding system is decreased from almost infinity to that of the mercury manometer, i.e., about $0.5 \mathrm{cc} / 20 \mathrm{~mm} \mathrm{Hg}$, while closure of the tubing at the point $\mathrm{Y}-\mathrm{Y}$ decreases the compliance almost to zero.

As is seen in FIG. 5, clamping at the point $\mathrm{X}-\mathrm{X}$ (indicated by arrow $\mathrm{A}$ ) at a moment when the SAP was reaching the downward peak of the waves caused 1) elevation of the mean SAP level by $10 \mathrm{~mm} \mathrm{Hg}$, and 2) increased peak to peak amplitude of the SAP waves because of the concomitant rise and fall of the side pressure owing to the now decreased compliance of the system. Further, clamping at the point $\mathrm{Y}-\mathrm{Y}$ at the similar moment (indicated by arrow B) caused an especially great rise in SAP which was not followed by any more oscillation. Contrary to this, when clamping was conducted at the same point during the SAP rise (indicated by arrow D), it resulted in the subsidance of oscillations with SAP remaining on the downward peak level this time. Such findings are also in good agreement with previous observations in rabbits $^{1,6)}$. 


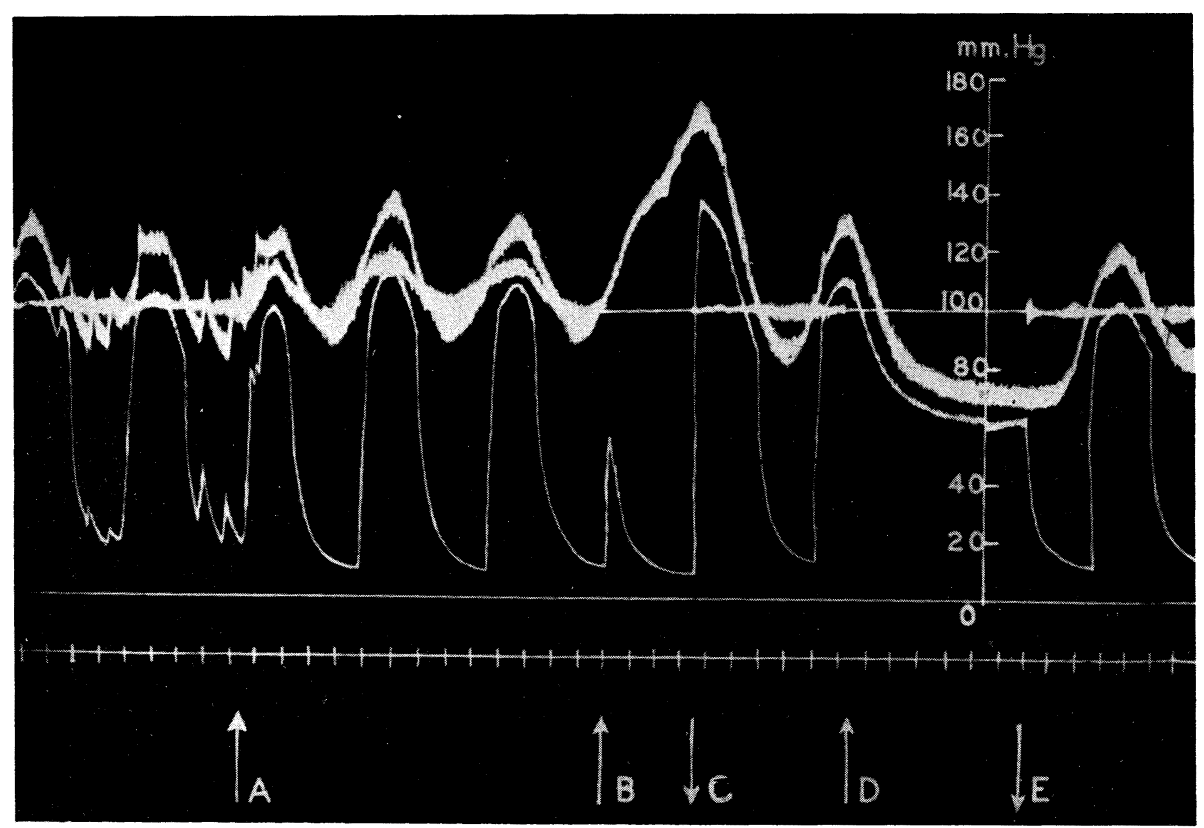

FIG. 5. Kymographic recording of the SAP, CPP, and SP. The side pressure loading rubber tubing was clamped at point $\mathrm{X}-\mathrm{X}$ (indicated by $\uparrow \mathrm{A}$ ), and at point $\mathrm{Y}-\mathrm{Y}$ when the collapsible tubing within the Starling's resistance had been closed (indicated by $\uparrow \mathrm{B}$ ) and patent (indicated by $\uparrow \mathrm{D}$ ). Note cessation of oscillations by clamping at point Y-Y. Refer to FIG. 1 as to the significance of clamping at these points. Time signal: 15 seconds.

\section{DISCUSSION}

Since the days of Traube and HERING, a number of experimental procedures and conditions have been reported to cause sustained oscillations of the mean arterial pressure level in varied experimental animals (for the review of historical literatures, see TIEGERSTADT ${ }^{\text {() }}$ ). In regard to the basic mechanism of such oscillations, some physiologists put emphasis upon the intrinsic trait of the circulatory center per se towards periodic undulation ${ }^{8-10)}$, while others are inclined to understand these phenomena as a sort of feedback oscillation elicited in blood pressure stabilizing mechanisms or in certain emergency reaction type mechanism ${ }^{11-14)}$.

Sustained oscillations of the arterial pressure were brought about in the present experiment by using essentially the same technique as MryaKAWA's regardless of the difference in experimental animals. This is undoubtedly because the oscillation is generated on the basis of cerebral ischemic pressor response mechanism which has been proved in many sorts of experimental animals. Having servoanalytic data on this basic response in dogs, the author 
seems to be in a more pertinent situation than the earlier investigators to discuss the mechanism of oscillation in favor of the feedback oscillation theory.

Concerning the stability (or condition for oscillation) in a negative feedback control system, NYQUIST found a criterion which would be abbreviated as follows; If the open loop over-all transfer function of a system has a gain factor over unity with a simultaneous phase lag factor of 180 degrees, the system will be quite unstable under closed loop conditions and is very likely to fall into feedback oscillation ${ }^{15}$. Based on this doctrine, the author investigated the stability of the cerebral ischemic pressor response regarding it as a negative feedback control mechanism of either the systemic arterial pressure, the cerebral blood flow, or the physico-chemical nature of cerebral interstitial fluid. As discussed in full detail in the previous report ${ }^{3)}$, the system was found to become quite unstable only if the mean level of the cerebral perfusion pressure was biased from the systemic arterial pressure down to such low values as 10 to $20 \mathrm{~mm} \mathrm{Hg}$. In other words, the mean cerebral perfusion pressure exerts a profound influence upon the system properties (parametric forcing) and renders the system very unstable as the CPP approaches zero, while the system remains quite stable as long as the CPP stays within physiological ranges. In addition, this theoretical outcome was satisfactorily confirmed by actual elicitation of the predicted feedback oscillation by the use of a servo-mechanism which does nothing but bias the mean cerebral perfusion pressure level from the systemic arterial pressure level by an arbitrarily set fixed magnitude ${ }^{4}$.

Now the question arises as to what condition made the whole system unstable enough to oscillate under the present circumstances. In order to answer this question, we simply have to recall the nonlinear transfer characteristic of the Starling's resistance demonstrated in FIG. 2. Working as a limiter for the CPP when the SP exceeds the SAP, and thus reducing the CPP toward zero in an exponential manner as evidenced in FIG. 3, it brings the mean value of the CPP's into a considerably lower range than that of the SAP's. Moreover, as shown again in FIG. 3, the resistance alters a small amount of time-dependent changes of the SAP above and below the SP into such large variations of the CPP that the degree of this nonlinear amplification amounts to as high as 2.5 to 12.0. Accordingly it is concluded that the Starling's resistance renders the system unstable in two ways, one biasing the operating range of the cerebral ischemic pressor response system towards an unstable region, i.e. through parametric forcing upon the responding element, and the other increasing directly the over-all system gain factor by its nonlinear amplification. On the other hand, the resistance causes an insignificant time lag as evidenced in FIG. 4, thus contributing little to the observed increase of system instability through phase lag factor. FIG. 6 explains the above mentioned considerations with a block diagram commonly used in control engineering. The block designated cerebral vascular conductance, circulatory center, and cardiovascular 


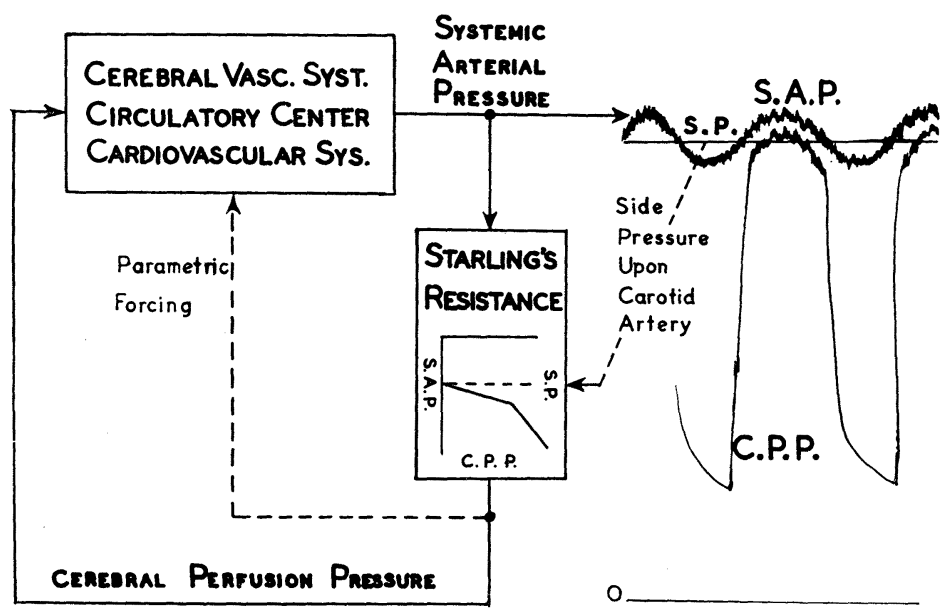

FIG. 6. Block diagrammatic explanation of the present experiment on the left half with tracing of kymographic record of systemic arterial pressure (SAP) and the cerebral perfusion pressure (CPP) distal to the Starling's resistance which was inserted in the common carotid artery to compress the latter with isotonic side pressure (SP) on the right half. Although the traditional term Starling's " resistance" is used here for easier understanding, the element should be preferably denoted as a dimensionless amplifier with limiter action. For further explanation, see text.

system denotes the entire physiological elements involved in the elicitation of cerebral ischemic pressor response. Under ordinary conditions, output of the block, i. e., the systemic arterial pressure, is fed directly back to its input side, since the cerebral perfusion pressure should be the systemic arterial pressure itself. In the present experiment, however, a Starling's resistance was inserted in the feedback loop, through which the average CPP level was biased toward zero and the time-dependent variable components of the SAP were strongly amplified in a non-linear fashion. Such modified pressure (CPP) was in turn fed back to the input side of the former block instead of the ordinary systemic arterial pressure (SAP). The parametric forcing which the low mean CPP exerts upon the system property is expressed by the broken line leading to the bottom of above mentioned block.

It seems to be of utmost importance to compare the nature of and the role played by the nonlinearity in the present experimental system with those in the previous study in which a feedback oscillation was brought about by inserting a bias-pressure generator between the SAP and the CPP. This biaspressure generator mechanism biases the mean CPP below the SAP by a desired constant amount but transfers the time dependent of the SAP to the CPP without either amplification or attenuation. In the present experiment the nonlinearity introduced by the Starling's resistance is postulated to constitute an essential factor for the genesis of oscillation as pointed out above, while in 
the previous experiment significant nonlinearity creeps into and modifies the oscillating process only after the amplitude of oscillation builds up to a certain magnitude. Accordingly, it will be clear now that the NYQUIST's theory, which may be applied only to a linear system, holds in the initial step of the genetic mechanism of oscillation in the previous experiment but does not in the present experiment because of the marked nonlinear operation of the Starling's resistance.

Nevertheless, the mechanism of oscillation in the present experiment will be explained without any difficulty as one of the relaxation oscillations which van der Pol discussed generally in mathematical terms ${ }^{16)}$. Closure of the cigarette skin tubing within the Starling's resistance due to the SAP's fall below the SP causes, an approximately exponential decrease in the CPP with a time constant dependent on the capacitive and resistive properties of the cerebral vascular system. This time constant appeared to be around 8 to 10 seconds in the present experiment. What curtails this exponential decrease is the cerebral ischemic pressor response and the resultant reopening of the Starling's resistance tubing as the SAP rises above the SP. The time course of this $\mathrm{CPP}$ rise depends again on the above mentioned time constant as well as on the intensity and the time course of the pressor response itself. Thus, it will be clear that the cerebral vascular time constant is a factor of major importance in deciding the period of oscillation reported here. The slope of the abruptly rising part of the pressure-flow curve through the Starling's resistance is another factor of definite importance. A strictly quantitative treatment of such factors can not be achieved here owing to the lack of accurate information on the dynamic performance of individual elements and to their nonlinearity in performance. Therefore, rather simplified considerations will be discussed here in view of the steady state response characteristics of the involved elements. FIG. 7 three sorts of interrelationships between the systemic arterial pressure and the cerebral perfusion pressure are expressed under three different conditions. The straight line a-a, starting from the origin with a slope of 45 degrees, shows the relationship between the two pressures under ordinary conditions. The second curve $b-b_{1}$ (or $b-b_{2}$ ) denotes the relationship when the Starling's resistance intervenes between the SAP and CPP as in the present experiment. It must be remembered that these curves are obtained when the SAP is varied primarily and the CPP changes as the dependent variable. The hyperbolic curve $c-c$, on the other hand, represents the relationship between two pressures obtained in the previous study in which the CPP was isolated from and changed independently of the SAP and the steady state response values of the SAP were plotted as the function of the given $\mathrm{CPP}^{2)}$. In other words, this curve indicates the steady state, open-loop response characteristics of the presumed negative feedback control of the SAP through the cerebral blood flow or the CPP. If one equates either one of the two former curves 


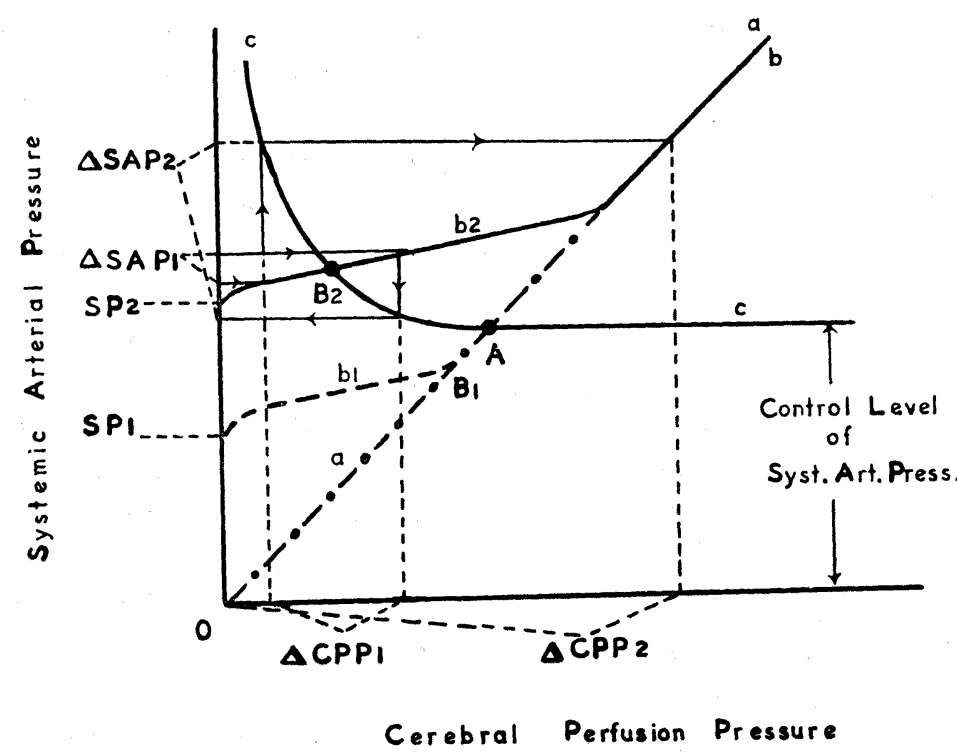

FIG. 7. Diagrammatic presentation of the steady state relationships between the SAP and CPP. Curve a-a, the straight line with a slope of 45 degrees, represents the relationship under normal conditions while the curves $b-b_{1}$ and $b-b_{2}$ indicate the relations when the Starling's resistance side pressure was set at $\mathrm{SP}_{1}$ and $\mathrm{SP}_{2}$ as shown on the ordinate (and thereby correspond to the pressure-flow curve in FIG. 2). In these two sorts of curves, the CPP's were plotted as a function of the SAP's. To the contrary, the relationship expressed by the hyperbolic curve $c-c$ was obtained when the CPP was primarily varied and the resultant SAP was plotted as its function (Ref. 2). Intersections of these curves, ( $A$ and $B_{1}$ ) demonstrates equilibrium point the system would attain under respective conditions, while the intersection $B_{2}$ indicates an equilibrium point which may actually not be reached because of the increased instability of the system. For further explanations, see text.

with the curve $c-c$, their intersection represents a steady state equilibrium point. For example, under ordinary conditions, the animal's SAP-CPP relationship ought to equilibriate at point $\mathrm{A}$ where the curves a-a and $\mathrm{c}-\mathrm{c}$ intersect. In the present experiment, however, the situation is different; curves $b-b_{1}, b-b_{2}$, etc. replacing curve $a-a$, the equilibrium point should be sought at $A_{1}, B_{2}$, etc. as the side pressure is raised at $\mathrm{SP}_{1}, \mathrm{SP}_{2}$, etc. An equilibrium presumed at point $B_{2}$ will be extremely unstable because with such low CPP's the ischemic response becomes highly sensitive, but at the same time tends to lag considerably in time in addition to the inherent dead time. With such delay and sensitivity in response increased beyond a critical amount, the system is likely to precipitate into an oscillatory state out of a slight inductive momentum. Although details of this oscillating mechanism can not be described without referring to the dynamic performance of each element of the 
response chain, instability of the system will be inferred from this graphical presentation of steady state response as follows: a small amount of change in the SAP $\left(\triangle \mathrm{SAP}_{1}\right.$ in FIG. 7$)$ above and below the point $\mathrm{B}_{2}$ will evoke much greater change in the CPP $\left(\triangle \mathrm{CPP}_{1}\right)$ than it would have elicited if the Starling's resistance had not been inserted and the CPP had changed along the curve $\mathrm{a}-\mathrm{a}$ instead of the curve $\mathrm{b}-\mathrm{b}_{2}$. The $\triangle \mathrm{CPP}_{1}$ will then cause, or at least tend to produce, $\triangle \mathrm{SAP}_{2}$ as determined by the curve c-c. The resultant $\triangle \mathrm{SAP}_{2}$, which is larger than the original change $\triangle \mathrm{SAP}_{1}$, will create another cycle of responses with the resultant $\triangle \mathrm{CPP}_{3}$, which is still larger than $\triangle \mathrm{CPP}_{1}$. This selfaugmenting cycle will keep growing in amplitude until the increase of $\triangle \mathrm{CPP}$ 's become restricted in one side by zero limit, and $\triangle$ SAP's increase is saturated at its lower margin on account of the less and less $\triangle \mathrm{SAP} / \triangle \mathrm{CPP}$ ratio toward higher CPP range along the curve c-c. These considerations on the steady state response diagram represent merely a gross approximation of the dynamic process of oscillating mechanism, and it should not be taken as the reality. As yet, it has a merit of intuitive clarity with which one can comprehend the genesis of oscillation in the present nonlinear system.

No detailed explanations will be needed now as to the reason why clamping the rubber tubing at the point $\mathrm{Y}-\mathrm{Y}$ in FIG. 5 ended the oscillation. The procedure alters the Starling's resistance into a more rigid system with the result that the SAP-CPP relationship curve across it approaches the curve a-a. What this alteration does for the oscillating process will be understood by referring to the above mentioned explanation. If clamping was performed at a moment when the cigarette skin tubing had been patent, response equilibrium would be reached in the neighbourhood of point $A$. If the tubing was clamped when the cigarette skin tubing had been closed, the response would advance toward the left upper end of curve c-c independently of the SAP rise. This is exactly what happened in these experiments as illustrated in FIG. 5.

Raising the intracranial pressure (ICP) by means of an isotonic system above the SAP level also causes sustained arterial pressure oscillations ${ }^{13}$. The present experiment may well be regarded as a modification of this long known experiment. On closer insight, however, two different situations can be pointed out as to the hemodynamics of cerebral circulation under these circumstances One is that collapse of the cerebral vascular system due to the elevated ICP presumably begins at the small veins within the subarachnoid space in the ICP elevation experiment, while it occurs at the carotid artery in the present experiment. Consequently, the cerebral blood flow will be cut off immediately after the vascular collapse occurs in the former case, while the blood flow continues for a while with exponential decrement in the present experiment. It must be this difference which led to the shorter period of pressure oscillation in the ICP elevation experiment $(16.8 \pm 4.8$ seconds in rabbits according to the author $\left.{ }^{6}\right), 25$ seconds and $32 \pm 12$ seconds in dogs according respectively to 
GUYTON $^{17)}$ and to the author ${ }^{18)}$ ) and to the longer period in experiments with side pressure load upon the carotid artery $(26 \pm 18.8$ seconds in rabbits according to MIYAKAWA ${ }^{19)}$ and 30 to 60 seconds in dogs in the present experiment).

Another possible difference which is principally interrelated with the first one is that, in the ICP elevation experiment, the cerebral arterial pressureblood flow relationship does not entertain any remarkable nonlinearity. This is a finding confirmed by NOEL and SCHNEIDER both in dogs and in a physical model, which is substantially similar to the Starling's resistance used in the present experiment except that they gave major flow impedance at the upstream side of the resistance so that the pressure in the collapsible tubing might simulate the low pressure in the cerebral vein ${ }^{20)}$. When they raised the chamber pressure (simulating ICP) step-wise with fixed perfusion (arterial) pressure, the flow volume decreased linearly. Consequently, one cannot expect such an exaggerated nonlinear amplification of the time dependent component in the SAP and thus a corresponding increase of over-all system gain as is the case with the SP loading experiment. In this respect, the mechanism of oscillation in ICP elevation experiment obviously differs from that revealed in the present study, and bears a closer analogy to the oscillation elicited in the CPP biasing experiment by the author in which nonlinearity is not essential for the initiation of oscillation.

\section{SUMMARY}

The effect of adding a side pressure of isotonic character upon the carotid artery, a method originally reported by MIYAKAWA to create sustained oscillations in the mean arterial pressure level of rabbits, was reinvestigated with minor technical modifications in dogs. The expected oscillation was brought about successfully in all dogs. By recording the cerebral perfusion pressure (i. e. the pressure at the point distal to the side pressure loading site) and the cerebral blood flow simultaneously with the systemic arterial pressure, the roles of the Starling's resistance used to add side pressure were clearly demonstrated: (1) it operates as a sort of limiter for cerebral perfusion pressure or blood flow, and thus amplifies the changes of the cerebral arterial pressure in a nonlinear fashion 2 to 12 times as large as that in the systemic arterial pressure, and (2) it also biases the mean operating level of the effective cerebral arterial pressure toward zero from the ordinary systemic arterial pressure level. Both roles increase the over-all gain and time lag of the system response and render the system quite unstable. Thus, the present experiment reveals the feedback nature of arterial pressure oscillation elicited by adding the side pressure upon the cerebral arterial pressure. 
The author wishes to thank Drs. Arthur C. Guyton and Ippei Hatakeyama for giving him the opportunity to conduct this experiment in the Department of Physiology and Biophysics, University of Mississippi Medical Center, and also for reading the manuscript for revision.

\section{REFERENCES}

1) Miyakawa, K. J. Physiol. Soc. Jap. 17: 299, 1955.

2) Sagawa, K., J. M. Ross, and A. C. Guyton. Amer. J. Physiol. 200 : 1164, 1961.

3) Sagawa, K., A. E. Taylor, and A. C. Guyton. Amer. J. Physiol. 201 : 1164, 1961.

4) Sagawa, K., O. Carrier, and A. C. Guyton. Amer. J. Physiol. 202 : 1962.

5) Sagawa, K., and A. C. Guyton. Amer. J. Physiol. $200: 711,1961$.

6) Sagawa, K. J. Physiol. Soc. Jap. $17: 402,1955$.

7) Tiegerstadt, R. Physiologie des Kreislaufes (2 Auflage). Berlin: Walter Furyter, 1923, Band 4, Seit 283.

8) Hartman, F. Z. Chir. $247: 242,1936$.

9) KNoll, P. Sitz. Kais. Akad. Abt. III $92: 439,1886$.

10) Wagner, R. und H. Schrocksandel. Methodik und Ergebnisse fortlaufender Blutdruckschreibung am Menschen. Leipzig: Thieme, 1942, Seit 63.

11) Anderson, B., R. A. Kenney, and E. Neil. Acta Physiol. Scand. 20 : 203, 1950.

12) Bauereisen, E., H. Drischel, H. Krug, U. Peiper, und L. Schlicher. Z. Biol. $110: 309,1958$.

13) Cushing, H. Johns Hopkins Hosp. Bull. 12: 290, 1901.

14) Guyton, A.C. Textbook of Med. Physiol. (2nd ed.) Philadelphia: Saunders, 1961, pp 8-12.

15) Truxal, J. G. Control Engineer's Handbook. New York; MacGraw-Hill, 1958, pp. $2-27$.

16) VAN DER PoL, B. Phil. Mag. 2: 978, 1926.

17) Guyton, A. C. and J. H. Satterfield. Amer. J. Physiol. 170: 601, 1952.

18) Sagawa, K. J. Physiol. Soc. Jap. 18: 508, 1956.

19) Miyakawa, K. J. Physiol. Soc. Jap. $17: 384,1955$.

20) Noel, W. und M. Schneider. Arch. Psychiat. 180: 713, 1948. 